\title{
EVOLUTION OF AN ACCRETION DISK IN THE SYMBIOTIC BINARY CI CYG
}

\author{
JOANNA MIKOLAJEWSKA \\ Copernicus Astronomical Center \\ Bartycka 18, PL-00716 Warsaw, Poland
}

\begin{abstract}
We have combined IUE spectra with optical spectroscopic and photometric data collected over a few orbits of the symbiotic binary CI Cyg to follow the evolution of a disk and boundary layer during a full activity cycle. Our results indicate an extended optically thin disk during quiescent periods, which evolves into an optically thick state in an eruption; a classical boundary layer at the inner edge of the disk ionizes a surrounding $\mathrm{H}$ II region in quiescence, and this emission fades during the rise to visual maximum in outburst. This evolution of $\dot{M}$ rivals that observed in classical CVs and premain sequence stars, so symbiotic systems like CI Cyg represent another opportunity to study the physics of disk accretion.
\end{abstract}

\section{Accretion models for symbiotic stars}

Symbiotic stars are commonly accepted as long period $\left(P_{\text {orb }} \sim 200 \ldots 1000 \mathrm{~d}\right)$ interacting binaries similar to cataclysmic variable stars. They consist of an evolved red giant star, a hot companion, and an ionized nebula. The hot components in many systems show occasional $2-3$ mag eruptions, characterized by dramatic changes of the optical spectrum. In particular, an $\mathrm{A}-\mathrm{F}$ supergiant star appears to replace the red giant at optical maximum and most high-ionization emission lines disappear. The cause of these eruptions remains controversial, as both accretion-powered events and thermonuclear runaways (TNR) in material accreted by a white dwarf can account for many features observed at a typical symbiotic outburst.

In accretion-powered systems, two physical mechanisms can change the accretion rate $(\dot{M})$ through an accretion disk, and as a result the hot component's luminosity; mass-transfer instabilities and disk instabilities (see e.g. Mikołajewska \& Kenyon 1992a for details). The 2-3 mag optical out- 
bursts require main sequence (MS) accretors (Bath \& Pringle 1982; Kenyon $\&$ Webbink 1984) unless $\dot{M}$ exceeds a value appropriate to the Eddington limit. Accretion-driven eruptions can be distinguished observationally from TNR events by an increase in the emitted flux at all wavelengths, rather than flux redistribution that characterizes the rise to optical maximum and early optical decline in the TNR case. MS accretors develop A-F supergiant optical spectra at maximum as do degenerate TNR events or, if $\dot{M}$ increases, steadily burning white dwarfs but the UV spectra more closely resemble late $\mathrm{B}$ or early $\mathrm{A}$ supergiants. This behavior is due to the radial temperature profile in the disk: $T_{\text {eff }}$ decreases from $\sim 20000 \ldots 30000 \mathrm{~K}$ in the inner disk to $\sim 5000 \ldots 10000 \mathrm{~K}$ in the outer disk. Finally, most disk models predict that a boundary layer (BL) between the disk and slowly rotating central star radiates $50 \%$ of the accretion energy. Since the BL temperature exceeds $\sim 10^{5} \mathrm{~K}$ for MS accretors, it emits mostly in the EUV range. This radiation photoionizes gas surrounding the disk, and possibly also the disk material, to produce optical and UV emission lines. The emission line fluxes provide a reasonably good estimate of the EUV luminosity, so the evolution of the BL can be determined with optical and UV spectra. An important prediction of the model is that the EUV luminosity from the BL should be comparable to the optical + UV luminosity from the disk.

\section{CI Cyg}

A good example of a symbiotic system with an accretion disk is CI Cyg, which underwent a major outburst in 1975 and recently returned to quiescence. The system contains an M5II giant, $M_{\mathrm{g}} \sim 1.5 \mathrm{M}_{\odot}$, transferring material into a large accretion disk around a low mass, $M_{\mathrm{h}} \sim 0.5 \mathrm{M}_{\odot}$, MS companion (Kenyon et al. 1991). The $U B V$ photometry (Belyakina 1991 and references therein) demonstrated that CI Cyg is an eclipsing system with $P_{\text {orb }}=855 \mathrm{~d}$. The characteristic double bump visible in the quiescent $V R I$ light curves (Belyakina 1992) indicates that the giant is tidally distorted, and that mass transfer occurs via Roche lobe outflow.

The early phases of the last eruption were observed only at optical and IR wavelengths, but the latter stages of the decline and the present quiescent phase were also well covered with the IUE. These data represent a unique opportunity to investigate the evolution of an accreting symbiotic system over a full activity cycle.

The 1975 outburst was characterized by the appearance of a bright optical continuum, increase of Balmer emission line fluxes, and the absence of high-ionization lines. The outburst also caused a significant change in the eclipses: they became narrow with well defined phases of contact, contrary to the broad minima observed at quiescence (Belyakina 1991; Kenyon et al. 
1991). The hot component's luminosity increased from $L_{\mathbf{h}} \sim$ a few $\times 10^{2} \mathrm{~L}_{\odot}$ at quiescence to $\sim 10^{4} \mathrm{~L}_{\odot}$ at maximum.

The optical colors at maximum were inconsistent with a single temperature stellar photosphere; the apparent spectral type varied from B7 $(U-B)$ through A5-A6 $(B-V)$ to F0 $(V-R$ and $R-I)$. During the late decline (1980), optical spectrophotometry indicated the presence of a totally eclipsed red continuum source $\left(T_{\mathrm{c}} \sim 4000 \mathrm{~K}, R \sim 50 \mathrm{R}_{\odot}\right.$; Mikołajewska \& Mikołajewski 1983), while the totally eclipsed short wavelength IUE continuum was consistent with a late B - early A spectral type. The eclipses allow us to estimate the outburst radius of the hot component in the orbital plane. This radius exceeds the spherically symmetric radius inferred from the total luminosity by a factor of $\sim 2 \ldots 3$. If the hot component is an ellipsoid, then the radius of the major axis exceeds the radius of the minor axis by a factor of $\sim 3-5$ (Mikołajewska \& Kenyon 1992a). The large values of $H / R \sim 0.2-0.3$ for the disk in CI Cyg agrees with Bath \& Pringle's (1982) predictions for high $-\dot{M}$ disks surrounding MS stars.

As the system declines from the visual maximum, strong high-ionization lines return. These emission lines require a source with a temperature of $\sim 10^{5} \mathrm{~K}$, and EUV luminosity comparable to the optical + UV luminosity. We associate this source with a boundary layer. Kenyon \& Webbink (1984; see also Plavec \& Hubeny 1994) fit the UV continuum at that time with optically thick steady-disk models, and these models suggest $\dot{M}$ declines with time from $\dot{M} \sim$ a few $\times 10^{-4}$ to a few $\times 10^{-5} \mathrm{M}_{\odot} \mathrm{yr}^{-1}$. The EUV luminosity estimated from strong $\mathrm{H}$ I, He I and He II emission lines follows the disk luminosity, with $L_{\mathrm{EUV}} \approx L_{\text {disk }}$ (Kenyon et al. 1991).

The eclipses in $\mathrm{HI}, \mathrm{He} \mathrm{I}$ and $\mathrm{He} \mathrm{II}$ emission lines are broad at quiescence and narrow during outburst, which indicates that the size of the $\mathrm{H} \mathrm{II}$ and He III region in the orbital plane shrinks in outburst (Mikołajewska 1985; Kenyon et al. 1991). Such behavior again points to the presence of an optically (and geometrically) thick accretion disk that prevents ionization in the orbital plane.

In 1983 CI Cyg entered the quiet phase. The color temperature of the short wavelength IUE continuum increased, while the long wavelength IUE and near UV continuum was dominated by strong bound-free + free-free emission. We interpret this as a transition from an optically thick to an optically (and geometrically) thin - at least in the optical range - disk. The UV continuum then became dominated by hot radiation from the boundary layer and the innermost accretion disk. The transition was also accompanied by development of strong high-ionization forbidden emission lines $[\mathrm{NeV}],[\mathrm{FeVII}]$ and $[\mathrm{Mg} \mathrm{V}]$. These forbidden lines cannot be produced in the same regions as the permitted emission lines, because they are not affected by the eclipses. In fact, the forbidden line ratios require lower densities, 
$n_{\mathrm{e}} \sim 10^{8} \mathrm{~cm}^{-3}$, and higher temperatures, $T_{\mathbf{e}} \sim 10^{5} \mathrm{~K}$, than the values, $n_{\mathrm{e}} \sim 10^{10} \mathrm{~cm}^{-3}, T_{\mathrm{e}} \sim 15000 \mathrm{~K}$, derived from the eclipsed permitted and intercombination lines (Kenyon et al. 1991). These results suggest a model in which the permitted and intercombination lines form in a photoionized region surrounding the hot component, and forbidden lines form in a larger shock-heated region produced by interacting winds or an accretion disk 'corona'.

\section{Concluding remarks}

The outburst behavior of CI Cyg - an increase in the emitted flux at all wavelengths, an ellipsoidal hot component, the variation of spectral type with wavelength - points to accretion-powered outburst with an MS accretor. The IUE and optical observations suggest that the disk and boundary layer each emit $\sim 50 \%$ of the total luminosity during the decline, which also agrees with simple disk models. There are however two deviations from the behavior predicted by theory: EUV emission from the BL vanishes near visual maximum, and a large mechanically heated region develops during the late decline from maximum. Similar behavior has occured in outbursts of AX Per, a 'sister' system of CI Cyg (Mikołajewska \& Kenyon 1992b). The disappearance of $\mathrm{BL}$ emission is probably related to changes in the physical conditions at the disk's inner edge as $\dot{M}$ increases in outburst. In fact, the BL emission initially tracks the rise in $\dot{M}$, but declines as $\dot{M}$ exceeds $\sim 10^{-4} \mathrm{M}_{\odot} \mathrm{yr}^{-1}$. Such behavior is also observed in pre-MS accretion disks: low $\dot{M}$ objects (T Tau stars) appear to have a standard BL, while in high $\dot{M}$ objects (FU Ori stars) the BL emission is missing (Kenyon 1994).

Acknowledgement. This study was supported by KBN Research Grant No. 2 P304 00706.

\section{References}

Bath, G. T., Pringle, J. E., 1982, MNRAS, 201, 345

Belyakina, T. S., 1991, Izv. Krym. Astrofiz. Obs., 83, 118

Belyakina, T. S., 1992, Izv. Krym. Astrofiz. Obs., 85, 62

Kenyon, S. J., 1994, in "Interacting Binary Stars", ed. A. Shafter, ASP Conf. Ser. 56, 63

Kenyon, S. J, Webbink, R. E., 1984, Ap. J., 279, 252

Kenyon, S. J., Oliversen, N. A., Mikolajewska, J., et al., 1991, AJ, 101, 637

Mikolajewska, J., 1985, Acta Astr., 35, 65

Mikolajewska, J., Kenyon, S. J., 1992a, MNRAS, 256, 177

Mikolajewska, J., Kenyon, S. J., 1992b, AJ, 103, 579

Mikolajewska, J., Mikołajewski, M., 1983, Acta Astr., 33, 403

Plavec, M. J., Hubeny, I., 1994, in "Interacting Binary Stars", ed. A. Shafter, ASP Conf. Ser. 56, 87 